\title{
Device Physics Analysis of Parasitic Conduction Band Barrier Formation in SiGe HBTs
}

\author{
K. P. Roenker* and S. A. Alterovitz** \\ - Department of Electrical and Computer Engineering and Computer Science \\ University of Cincinnati, Cincinnati, Ohio 45221-0030 \\ Tel: (513)-556-4761 Fax: (513)-556-7326 Email: kroenker@ececs.uc.edu \\ **NASA Glenn Research Center, Cleveland, Ohio 44135 Email: salterovitz@grc.nasa.gov
}

\begin{abstract}
This paper presents a physics-based model describing the current-induced formation of a parasitic barrier in the conduction band at the basecollector heterojunction in npn SiGe heterojunction bipolar transistors (HBTs). Due to the valence band discontinuity $\Delta \mathbf{E}_{\mathrm{v}}$, hole injection into the collector at the onset of base pushout is impeded, which gives rise to formation of a barrier to electron transport which degrades the device's high frequency performance. In this paper, we present results from an analytical model for the height of the barrier calculated from the device's structure as a function of the collector junction bias and collector current density.
\end{abstract}

\section{INTRODUCTION}

In recent years SiGe heterojunction bipolar transistors (HBTs) have been reported with high gain and impressive high frequency performance $[1,2]$. These devices employ $\mathrm{Ge}$ in the base and compositional base grading so that a heterojunction is formed at the collector junction. While the conduction band discontinuity is small, the presence of a valence band discontinuity $\Delta E_{v}$ at the junction modifies the physics of electron transport across the collector junction at high current densities. Dynamic formation of a parasitic barrier in the conduction band occurs, which degrades device performance [3-5]. The effect is important for device design since transistor operation at high collector current densities ( $\mathrm{J}_{\mathrm{C}}$ $\sim \operatorname{lmA} / \mu \mathrm{m}^{2}$ ) is essential to achieve high gain at microwave frequencies. Previously, Joseph et al. [6] employed a numerical simulator and showed that a parasitic barrier as large as $34 \mathrm{meV}$ forms at current densities of $\sim 4 \mathrm{~mA} / \mu \mathrm{m}^{2}$. Song and Yuan [7] and Mazhari and Morkoc [8] have reported simple, physics-based models to describe the formation of this parasitic barrier predicting similar and much larger barrier heights, respectively. The motivation for this study is to develop an enhanced description of the physics of this barrier formation for use in device design.

\section{ANALYTICAL MODEL}

Shown in Figure 1 is a schematic profile of the electric field assumed at the base-collector junction during formation of the parasitic barrier. This profile is similar to that reported by Joseph et al. [6], which they derived from their numerical simulator. Due to device operation at high current density, the peak field is pushed to the subcollector interface corresponding to the onset of base pushout. However, for SiGe HBTs, the valence band discontinuity at the base-collector junction blocks hole flow out of the base. As a result, a current-induced, parasitic barrier forms near the junction corresponding to a positive field region. This barrier inhibits electron injection into the collector from the base producing electron buildup at the end of the base, increased base recombination and degradation in the current gain.

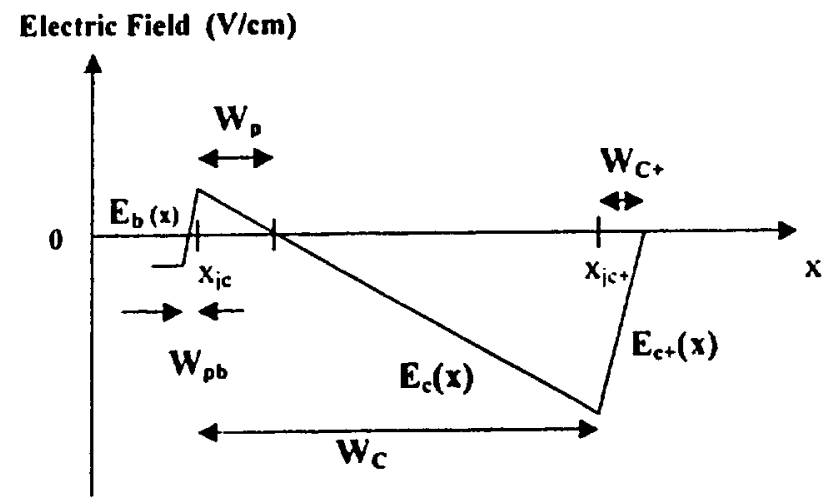

Figure I Electric field profile at B-C junction at high current density during formation of parasitic barrier.

For development of this device model, we note that the electron concentration $n_{3}$ is nearly constant in the high electric field in the base-collector space charge region (BC-SCR) near the subcollector due to velocity saturation $v_{s}$. As a result, the electron concentration is given by $n_{3}=$ $\mathrm{J}_{\mathrm{C}} / \mathrm{qv}_{3}$, where $\mathrm{J}_{\mathrm{C}}$ is the collector current density and the term arises from the need for a finite electron concentration sufficient to carry the collector current. Substituting this $n_{3}$ in Poisson's equation, we integrate to get the electric field in the depleted collector $E_{c}(x)$, which varies linearly as shown in Figure 1. On the base side of the heterojunction, we note that we have majority carrier hole accumulation due to the valence band discontinuity $\Delta E_{V}$, which we take into account in determining the electric field in the base $E_{b}(x)$. The location where the electric field is zero in the collector corresponds to the peak of the parasitic potential barrier.

To analyze the formation of the barrier, we initially match the fields at the base-collector and subcollector junctions. Subsequently, we piecewise integrate the electric field across the junction to relate our results to the collector doping and width and the applied junction 
reverse bias $V_{C B}$. Combining these results, we get a single equation for the potential at the base-collector junction $\psi\left(\mathrm{x}_{\mathrm{jc}}\right)$, where our zero for the potential was taken to be in the quasi-neutral base. After simplification, the solution is expressed in terms of the function $f\left(\psi\left(x_{j e}\right)\right)$ as

$$
f(\psi)=\frac{\mathcal{P}_{k}^{2}}{2 J_{b}\left[J_{b}+J_{C}-J_{1}\right]}
$$

where $J_{1}=q N_{C} v_{3}, J_{1+}=q N_{C+} v_{3}, J_{b}=q N_{B} v_{3}$, and $f\left(\psi\left(x_{j c}\right)\right)$ is given by

$$
\left.f\left(\psi\left(x_{j k}\right)\right)=\sqrt{\frac{2 \varepsilon V_{I}}{q N_{B} W_{C}^{2}}\left\{e^{-\lambda\left(x_{j}\right) V_{T}}-1+\frac{\left(\psi\left(x_{j c}\right)\right.}{V_{T}}\right.}\right\}
$$

where $J_{k}$ is given by

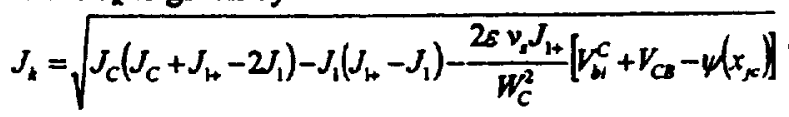

From (1)-(3) it is apparent that $\psi\left(x_{j c}\right)$ is a function of the device structure, current density $\mathrm{J}_{\mathrm{C}}$ and $\mathrm{V}_{\mathrm{CB}}$. From (3), we see that $J_{k}$ is nearly independent of $\psi\left(x_{j e}\right)$ so we can neglect it in calculating $J_{k}$, find $f(\Psi)$ from (1), and then determine $\psi\left(\mathrm{x}_{\mathrm{j}}\right)$ from (2). We can then determine the height of the parasitic potential barrier $\phi_{b}$ from the expression below, which corresponds to the magnitude of the potential at the point where the electric field in the depleted collector $E_{c}\left(x_{j c}+W_{p}\right)$ is equal to zero.

$$
\phi_{b}\left(J_{c}\right)=\frac{W_{C}^{2}}{2 \varepsilon v_{s}} \frac{J_{b}^{2} f^{2}\left(\psi\left(x_{j c}\right)\right)}{J_{c}-J_{1}}
$$

\section{SIMULATION RESULTS}

The above described device model was used to investigate the extent of the formation of the parasitic barrier $\phi_{B}$ and base pushout $W_{\text {cib }}$ for a typical device structure similar to that of Joseph et al. [6]. Linear compositional grading from zero at the emitter to $10 \% \mathrm{Ge}$ at the collector end of the base was assumed corresponding to $\Delta \mathrm{E}_{\mathrm{V}}=75 \mathrm{meV}$ at the collector junction. A base width of $90 \mathrm{~nm}$ was assumed with a doping of $1 \times 10^{18} / \mathrm{cm}^{3}$. A collector width $W_{c}$ of $0.5 \mu \mathrm{m}$ and doping of $1 \times 10^{17} / \mathrm{cm}^{3}$ was used. The current density constants $J_{1}$, $J_{1+}$ and $J_{b}$ were calculated to be $1.6,160$, and $16 \mathrm{~mA} / \mu \mathrm{m}^{2}$, respectively. A builtin potential of $0.75 \mathrm{~V}$ and a junction reverse bias of $1 \mathrm{~V}$ were assumed.

Shown in Figure 2 is the parasitic barrier $\phi_{b}$ plotted as a function of the collector current density. The onset of formation of the parasitic barrier $\phi_{0}$ occurs at a current density of $1.75 \mathrm{~mA} / \mu \mathrm{m}^{2}$, which is slightly larger than $J_{1}=$ $1.6 \mathrm{~mA} / \mathrm{mm}^{2}$. The parasitic barrier shows a sharp increase with increasing $\mathrm{J}_{C}$, which is comparable to that described by Mazhari and Morkoc [8], but larger than that reported by Joseph et al. [6] and Song and Yuan [7].

Since the formation of this parasitic barrier leads to excess electron buildup at the collector end of the quasineutral base, it produces a saturation effect in the collector current and an increase in the quasi-neutral base recombination, with a corresponding falloff in the current gain. This also degrades the base transit time and the cutoff frequency so that delay of the phenomena to higher

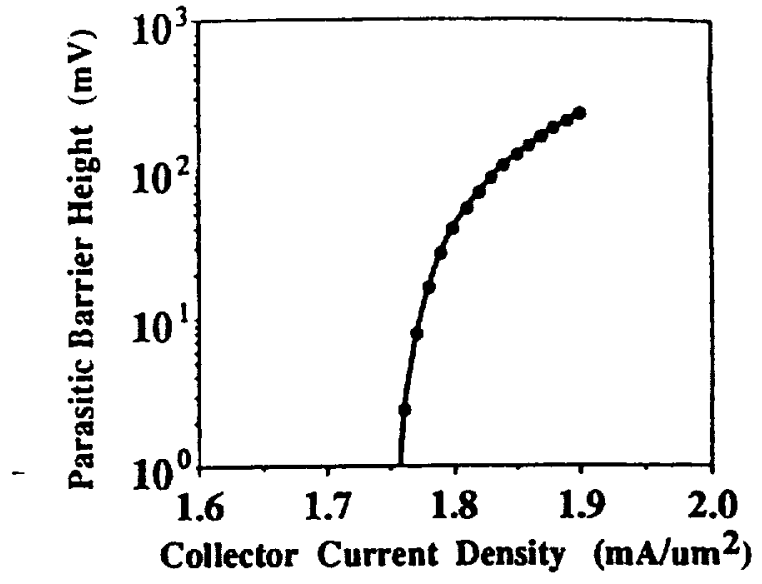

Figure 2 Parasitic barrier beight h as a function of collector current density.

current densities is desirable. Increasing the collector junction reverse bias and the collector doping help in this regard.

\section{CONCLUSIONS}

In summary, we have developed an improved description of the physics associated with the onset of the dynamic formation of the parasitic barrier at the basecollector junction at high collector current densities. The model will provide a useful tool for device engineers in the design of the base-collector junction for optimizing the device's performance at high current densities near the onset of base pushout.

\section{ACKNOWLEDGEMENTS}

This work was supported by a NASA Summer Faculty Fellowship from the Glenn Research Center.

\section{REFERENCES}

1. J. D. Cressler, "SiGe HBT Technology: A New Contender for SiBased RF and Micrownve Circuit Applications," IEEE Trans. Microwave Theary Tech, 46, 572, May 1998.

2. U. Konig "SiGe \& GaAs as Competitive Technologies for RF. Applications," Proc. IEEE Bipolar/BKCMOS Circuits Technol. Meeting, 1998, 87.

3. S. Tiwari, "A New Effect at High Currents in Heterostructure Bipolar Trensitose, "IEEE Electron Device Lett. 9, 142, Mrr. 1988.

4. J. W. Slotboom, O. Streutker, A. Pruifmboom and D. J. Gravesteijn, "Parasitic Enerey Buriers in SiGe HBT" \&" IEEE Elcetron Device Let. 12, 486, Seph 1991.

5. P. E. Coutrell and Z. Yu "Velocity Saturntion in the Collector of Si/GeSiSi HBT \&" IEEE Electron Drvice Lett 10, 431, Oct. 1990.

6. A. J. Joseph, J. D. Creasler, D. M. Richey and G. Nlu, "Optimization of SiGe HBT"s for Operntion at High Current Densities," IEEE Trans, Electron Devices 46, 1347, July 1999.

7. J. Song and J. S. Yuan, "Modelling the Baso-Collector Heterojunction Berrier Effect at Hifh Currem Dewaities," Solld State Electron 43, 457,1999.

8. B. Mreheri and H. Mottoc, Tefiect of Collector-Buse Valance Band Discontinuity on Kitk Effect in Doubls Heterojunction Bipolar Transistors," Appl. Physe Lets S3, 2162, Oct 1991. 


\title{
Device Physics Analysis of Parasitic Conduction Band Barrier Formation in SiGe HBTs
}

\author{
K. P. Roenker* and S. A. Alterovitz** \\ - Department of Electrical and Computer Engineering and Computer Science \\ University of Cincinnati, Cincinnati, Ohio 45221-0030 \\ Tel: (513)-556-4761 Fax: (513)-556-7326 Email: kroenker@ececs.uc.edu \\ * NASA Glenn Research Center, Cleveland, Ohio 44135 Email: salterovitz@grc.nasa.gov
}

\begin{abstract}
This paper presents a physics-based model describing the current-induced formation of a parasitic barrier in the conduction band at the basecollector heterojunction in npn SiGe heterojunction bipolar transistors (HBTs). Due to the valence band discontinuity $\Delta \mathbf{E}_{\mathrm{V}}$, hole injection into the collector at the onset of base pushout is impeded, which gives rise to formation of a barrier to electron transport which degrades the device's high frequency performance. In this paper, we present results from an analytical model for the height of the barrier calculated from the device's structure as a function of the collector junction bias and collector current density.
\end{abstract}

\section{INTRODUCTION}

In recent years $\mathrm{SiGe}$ heterojunction bipolar transistors (HBTs) have been reported with high gain and impressive high frequency performance [1,2]. These devices employ $\mathrm{Ge}$ in the base and compositional base grading so that a heterojunction is formed at the collector junction. While the conduction band discontinuity is small, the presence of a valence band discontinuity $\Delta \mathrm{E}_{\mathrm{V}}$ at the junction modifies the physics of electron transport across the collector junction at high current densities. Dynamic formation of a parasitic barrier in the conduction band occurs, which degrades device performance [3-5]. The effect is important for device design since transistor operation at high collector current densities $\left(\mathrm{J}_{\mathrm{C}}\right.$ $\sim \operatorname{lmA}\left(\mu \mathrm{m}^{2}\right)$ is essential to achieve high gain at microwave frequencies. Previously, Joseph et al. [6] employed a numerical simulator and showed that a parasitic barrier as large as $34 \mathrm{meV}$ forms at current densities of $\sim 4 \mathrm{~mA} / \mu \mathrm{m}^{2}$. Song and Yuan [7] and Mazhari and Morkoc [8] have reported simple, physics-based models to describe the formation of this parasitic barrier predicting similar and much larger barrier heights, respectively. The motivation for this study is to develop an enhanced description of the physics of this barrier formation for use in device design.

\section{ANALYTICAL MODEL}

Shown in Figure 1 is a schematic profile of the electric field assumed at the base-collector junction during formation of the parasitic barrier. This profile is similar to that reported by Joseph et al. [6], which they derived from their numerical simulator. Due to device operation at high current density, the peak field ${ }^{-}$is pushed to the subcollector interface corresponding to the onset of base pushout. However, for SiGe HBTs, the valence band discontinuity at the base-collector junction blocks hole flow out of the base. As a result, a current-induced, parasitic barrier forms near the junction corresponding to a positive field region. This barrier inhibits electron injection into the collector from the base producing electron buildup at the end of the base, increased base recombination and degradation in the current gain.

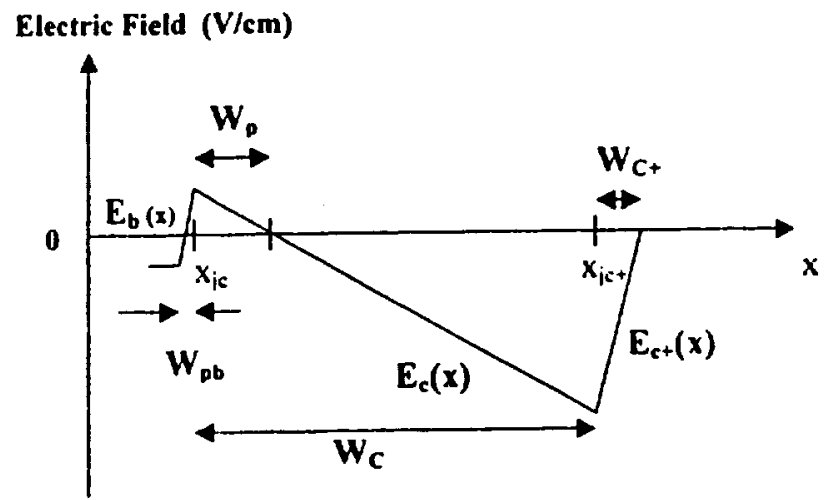

Figure 1 Electric field profile at B-C junction at high current density during formation of parasitic barrier.

For development of this device model, we note that the electron concentration $n_{3}$ is nearly constant in the high electric field in the base-collector space charge region (BC-SCR) near the subcollector due to velocity saturation $v_{3}$. As a result, the electron concentration is given by $n_{3}=$ $\mathrm{J}_{\mathrm{C}} / \mathrm{qv}_{\mathrm{s}}$, where $\mathrm{J}_{\mathrm{C}}$ is the collector current density and the term arises from the need for a finite electron concentration sufficient to carry the collector current. Substituting this $n_{3}$ in Poisson's equation, we integrate to get the electric field in the depleted collector $E_{c}(x)$, which varies linearly as shown in Figure 1 . On the base side of the heterojunction, we note that we have majority carrier hole accumulation due to the valence band discontinuity $\Delta E_{V}$, which we take into account in determining the electric field in the base $E_{b}(x)$. The location where the electric field is zero in the collector corresponds to the peak of the parasitic potential barrier.

To analyze the formation of the barrier, we initially match the fields at the base-collector and subcollector junctions. Subsequently, we piecewise integrate the electric field across the junction to relate our results to the collector doping and width and the applied junction 
reverse bias $\mathrm{V}_{\mathrm{CB}}$. Combining these results, we get a single equation for the potential at the base-collector junction $\psi\left(x_{j c}\right)$, where our zero for the potential was taken to be in the quasi-neutral base. After simplification, the solution is expressed in terms of the function $f\left(\psi\left(x_{j c}\right)\right)$ as

$$
f(\psi)=\frac{\overrightarrow{F_{k}}}{2 J_{b}\left[J_{H}+J_{C}-J_{1}\right]}
$$

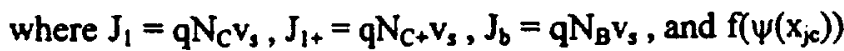
is given by

$$
f\left(\psi\left(x_{j s}\right)\right)=\sqrt{\frac{2 \varepsilon V_{T}}{q N_{B} W_{C}^{2}}\left\{e^{-\psi\left(x_{s}\right) V_{T}}-1+\frac{\left(\psi\left(x_{j s}\right)\right.}{V_{T}}\right\}}
$$

where $J_{k}$ is given by

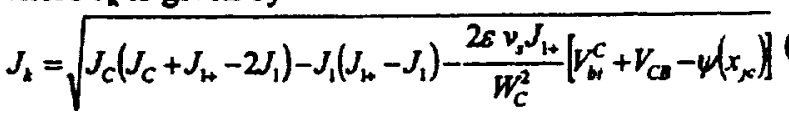

From (1)-(3) it is apparent that $\psi\left(x_{j e}\right)$ is a function of the device structure, current density $J_{C}$ and $V_{C B}$. From (3), we see that $J_{k}$ is nearly independent of $\psi\left(x_{j c}\right)$ so we can neglect it in calculating $J_{k}$, find $f(\psi)$ from (1), and then determine $\psi\left(\mathrm{x}_{\mathrm{je}}\right)$ from (2). We can then determine the height of the parasitic potential barrier $\phi_{b}$ from the expression below, which corresponds to the magnitude of the potential at the point where the electric field in the depleted collector $E_{c}\left(x_{j c}+W_{p}\right)$ is equal to zero.

$$
\phi_{b}\left(J_{c}\right)=\frac{W_{c}^{2}}{2 \varepsilon v_{s}} \frac{J_{b}^{2} f^{2}\left(\psi\left(x_{j c}\right)\right)}{J_{c}-J_{1}}
$$

\section{SIMULATION RESULTS}

The above described device model was used to investigate the extent of the formation of the parasitic barrier $\phi_{\mathrm{B}}$ and base pushout $W_{\mathrm{cib}}$ for a typical device structure similar to that of Joseph et al. [6]. Linear compositional grading from zero at the emitter to $10 \% \mathrm{Ge}$ at the collector end of the base was assumed corresponding to $\Delta E_{Y}=75 \mathrm{meV}$ at the collector junction. A base width of $90 \mathrm{~nm}$ was assumed with a doping of $1 \times 10^{18} / \mathrm{cm}^{3}$. A collector width $W_{c}$ of $0.5 \mu \mathrm{m}$ and doping of $1 \times 10^{17} / \mathrm{cm}^{3}$ was used. The current density constants $\mathrm{J}_{1}$, $J_{1}$ and $J_{b}$ were calculated to be $1.6,160$, and $16 \mathrm{~mA} / \mu \mathrm{m}^{2}$, respectively. A builtin potential of $0.75 \mathrm{~V}$ and a junction reverse bias of $1 \mathrm{~V}$ were assumed.

Shown in Figure 2 is the parasitic barrier $\phi_{b}$ plotted as a function of the collector current density. The onset of formation of the parasitic barrier $\phi_{b}$ occurs at a current density of $1.75 \mathrm{~mA} / \mu \mathrm{m}^{2}$, which is slightly larger than $J_{1}=$ $1.6 \mathrm{~mA} / \mu \mathrm{m}^{2}$. The parasitic barrier shows a sharp increase with increasing $\mathrm{J}_{\mathrm{C}}$, which is comparable to that described by Mazhari and Morkoc [8], but larger than that reported by Joseph et al. [6] and Song and Yuan [7].

Since the formation of this parasitic barrier leads to excess electron buildup at the collector end of the quasineutral base, it produces a saturation effect in the collector current and an increase in the quasi-neutral base recombination, with a corresponding falloff in the current gain. This also degrades the base transit time and the cutoff frequency so that delay of the phenomena to higher

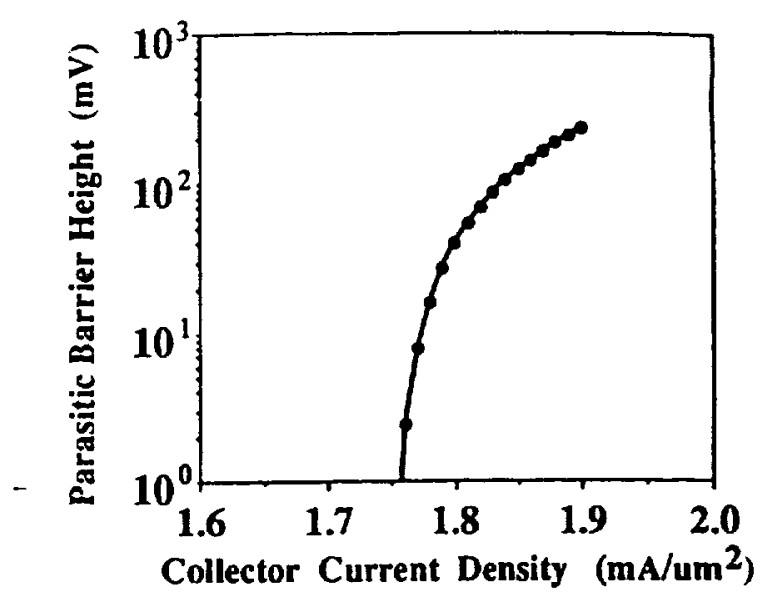

Figure 2 Parasitic barrier beight as a function of collector current density.

current densities is desirable. Increasing the collector junction reverse bias and the collector doping help in this regard.

\section{CONCLUSIONS}

In summary, we have developed an improved description of the physics associated with the onset of the dynamic formation of the parasitic barrier at the basecollector junction at high collector current densities. The model will provide a useful tool for device engineers in the design of the base-collector junction for optimizing the device's performance at high current densities near the onset of base pushout.

\section{ACKNOWLEDGEMENTS}

This work was supported by a NASA Summer Faculty Fellowship from the Glenn Research Center.

\section{REFERENCES}

1. J. D. Cressler, "SiGe HBT Technology: A New Contender for SiBased RF and Microwave Circuit Applications," IEEE Trans. Microwave Theory Tech, 46, 572, May 1998.

2. U. Konig "SiGe GaAs as Competitivo Technologies for RFApplications," Proc. IEEE Bipolar/BICMOS Circuils Technol. Meeting, 1998, 87.

3. S. Tiwari, "A New Effect at High Currents in Heterostructure Bipolar Transistors," IEEE Electron Device Leth. 9, 142, Mar. 1988.

4. I.W. Slotboom, G. Streutker, A. Pruijmboom and D. J. Gravesteijn, "Parasitic Energy Berriers in SiGe HBT's," IEEE Electron Device Lett. 12, 486, Sept. 1991.

5. P. E. Cottrell and Z. Yu, "Velocity Saturation in the Collector of Si/GeSi/Si HBT's," IEEE Electron Device Letr. 10, 431, Oct. 1990.

6. A. J. Joseph, J. D. Cressler, D. M. Richey and G. Niu, "Optimization of SiGe HBT's for Operation at High Current Densities," IEEE Trans. Electron Devices 46, 1347, July 1999.

7. J. Song and J. S. Yuan, "Modeling the Base-Collector Heterojunction Barrier Efiect at High Current Densities," Solld State Electron 43, 457,1999.

8. B. Mratheri and H. Morkoc, "Effect of Collector-Base Valance Band Discontinuity on Kirk Effect in Double Heterojunction Bipolar Transistors," Appl. Phys. Leth 59, 2162, Oct. 1991. 